\title{
Development of a National Student Mental Health and Suicide Prevention Framework for Higher Education in Ireland
}

\author{
Treasa Fox, Deirdre Byrne, Jessica Surdey \\ The SynthSCS Project \\ Athlone Institute of Technology \\ Ireland
}

\begin{abstract}
A focus of the SynthSCS project was to assist the Higher Education Authority in supporting Ireland's National Strategy to Reduce Suicide across all Higher Education Institutions. In order to meet these goals, a national framework guide for student mental health and suicide prevention was developed. The National Student Mental Health and Suicide Prevention Framework [1] [this framework] was the product of a collaborative cross disciplinary team including health professionals, government representatives, educators, students, policy makers, community organizations, researchers and clinicians. The mental health of students in higher education has become a major global concern. Students are identified as a highrisk group for mental health difficulties and are at greater risk of suicide. Concern for the increased levels of student mental illness, mental distress and low wellbeing has been recognized by the World Health Organization [2], My World-2: National Study of Youth Mental Health in Ireland [3] [MWS2], My World Survey: National Study of Youth Mental Health in Ireland [4] [MWS-1] and the Union of Students in Ireland National Report on Student Mental Health in Third Level Education [5]. This framework is informed by international evidence that calls for an embedded whole system approach to student metal health and well-being. The framework details nine interconnected themes and is endorsed by key stakeholders, launched on World Mental Health Day October 10th 2020 and is expected to be translated into National policy and practice. The framework has the potential to encourage the adoption of a national whole systems approach to student mental health and suicide prevention that is embedded in campus culture across higher education in Ireland and beyond.
\end{abstract}

\section{Introduction}

The SynthSCS Project - knowledge transfer and synthesis across student counselling services was established in 2019 and is funded by Higher Education Authority [HEA] Innovation and Transformation Fund 2018. The HEA is the statutory policy-advisory body for higher education in Ireland, providing policy-advice across all aspects of higher education. One of the aims of the project was to assist the HEA in fulfilling its commitment to the Department of Health's Connecting for Life Ireland's National Strategy to Reduce Suicide 20152020 to develop national guidelines for higher education in relation to suicide risk and critical incident response [6]. Young adults are identified as a group who are at increased risk of suicide and the majority of third-level students fall within this group.

To meet the goal of developing national guidelines, the HEA formed a working group on suicide prevention in higher education in 2018. Members of the working group were drawn from the HEA, the Health Service Executive's National Office of Suicide Prevention, the Union of Students in Ireland, Psychological Counsellors in Higher Education Ireland; Irish Students Health Association; National Suicide Research Foundation, as well as nominees from the Irish Universities Association, the Technological Higher Education Association, Mental Health Ireland, the Irish Research Council, the Department of Education and Skills, and the Mental Health Division of the HSE.

From the outset the working group took the view that suicide prevention in higher education could not be viewed as a stand-alone issue and must sit within wider national guidelines for student mental health, so the scope of the framework was expanded to cover student mental health and suicide prevention. The resulting national framework for Ireland's higher education system was developed and aims to address the issues of student mental health and suicide prevention in a structured and planned way.

\section{Background}

Over the past decades there has been a steady increase in the proportion of school leavers entering higher education: from $10 \%$ in the mid-1960s to $65 \%$ of the relevant age cohort in 2010 [7]. The most recent figures show that there are 238,555 students enrolled across the higher education system [8], [9].

This widening participation has resulted in more students from increasingly diverse backgrounds including under-represented groups, economically disadvantaged, mature students and students with disabilities. While the majority of students cope well 
in higher education, some groups and demographics do not. Some of the factors that may increase the risk of mental health difficulties for students have been identified and include: academic pressures; exam and assignment stress; transitions in and out of higher education; financial burdens; managing jobs and academic work; social and cultural pressures that include family, friends and intimate relationships; social media; and broader geopolitical concerns.

Some groups of students are at higher risk of experiencing mental health difficulties including: those who identify as LGBTQ+; international students; asylum seekers and refugees; those who have experienced trauma; online/remote students; first generation students; mature students; those from disadvantaged socioeconomic backgrounds, and those from ethnic minorities [3]. Students at increased risk of suicide, including those who self-harm; those who have been bereaved by suicide; those who have existing health or psychological conditions or difficulties; and those who have a history of drug or alcohol misuse [10].

Table 1. Numbers at a glance
Globally
- $75 \%$ of serious mental health difficulties emerge in the age range $15-25$ (Kessler et al., 2005)
- $35 \%$ of 1 st year students screened positive for at least one mental health disorder - depression, anxiety, substance use, etc. (WHO, 2018)
Suicide is a leading cause of death of young people (WHO, 2018)
Irish mental health
- $18.5 \%$ of adults have a mental health disorder (OECD, 2018)
v $€ 8.2$ billion annual costs arise from those mental health disorders (OECD, 2018)
- 20-24 year olds have the highest rate of inpatient psychiatric admissions (HRB, 2019)

\section{Irish higher education students}

- $6 \%$ of adults in Ireland are studying in HEls (HEA, 2018)

- 231,710 students are enrolled (HEA, 2019)

\section{Irish higher education student mental health}

- $127 \%$ increase in students registered with disability services for mental health over the past 5 years (AHEAD, 2019)

- $21 \%$ of respondents fell into the severe and very severe categories for depression (MWS 2) v $14 \%$ (MWS 1)

n $26 \%$ of respondents fell into the severe and very severe categories for anxiety (MWS 2) v15\% (MWS 1)

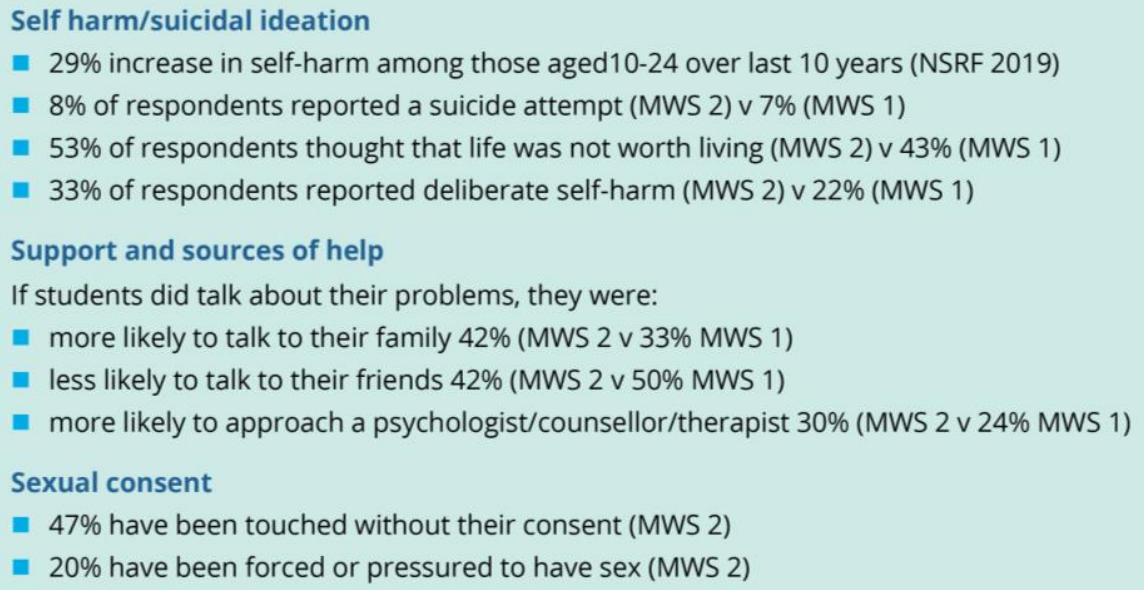

Ireland has one of the highest rates of mental illness in Europe (see table 1 above), with $18.5 \%$ of the population recorded as having a mental health condition [11]. It can therefore be expected that a 
significant number of students enrolled in Ireland's HEIs will have mental health issues.

Almost $75 \%$ of all serious mental health conditions first emerge between the ages of 15 and 25 [12], majority of full-time undergraduate entrants fall within that age group [13]. The college age population is identified as a vulnerable group by the National Office of Suicide Prevention and recent surveys of young people in Ireland recognize that young people are struggling with mental health [3] and internationally the World Mental Health International College Student Initiative [2], found that in higher education across the world demand far exceeds the resources available.

Higher education in Ireland is provided by universities, technological universities, institutes of technology, colleges of education and other higher education institutes, with populations ranging from 1,000 to 30,000 students. Increased access and widening participation in higher education has resulted in more students from more diverse backgrounds who may require robust support from institutions to foster student success.

While many HEIs provide guidance, policies and procedures and practical support, not all have fully documented guidance on areas such as mental health policy, critical incident procedures, distressed student protocols, student death policy, and the use of emergency response teams. Where guidance does exist, it is not always clear how it is implemented in practice or embedded in campus life.

HEIs provide students with a range of support services that includes counselling, access, tutors/help centers, disability services, health centers, students' unions, chaplaincy, careers advisory service, and academic writing support. While mental health first responder resources on campus can include any staff in a student-facing role, the increasing demand for professional supports for student mental health is largely provided by student counselling services, disability support services and student health centers. The demand for support services are growing. Over the last five years, there has been a $127 \%$ increase in students registered with disability support services for mental health conditions [14].

\section{Methods}

The overall goal of the SynthSCS project was to gain an understanding of student mental health and suicide prevention in higher education in Ireland. The project centred on four main activities, a scoping review of practices, initiatives and resources in Higher Education Institutions [HEI] student counselling services in Ireland and best practice internationally, assisting the HEA in developing national guidance to reduce suicide risk in higher education, forming partnerships/collaborative networks across sectors and disciplines, and create an online student mental health resource repository. This article concentrates on the background literature review that identified international best practices in student mental health.

The literature reviewed for this study looked at how student mental health and suicide prevention is addressed in Ireland and internationally. The search terms and key words included: student mental health, student wellbeing, institution student mental health supports, higher education mental health policy, suicide prevention in higher education, and national student mental health policy. The search was limited to literature published within the last fifteen years, in the English language, on student mental health in the higher education/third level/university sector. The databases searched systematically were EBSCO, Behavioural and Social Sciences, MEDLINE, PsycINFO, Scopus and Web of Science also included were government, health and other policy documents.

A critical review and analysis of the literature provided an overview of the knowledge base, with the aim of expanding the theoretical foundation of this topic. This knowledge was developed to create the building blocks of the national framework for student mental health and suicide prevention in higher education in Ireland by providing an evidence base and guidelines for policy and practice.

The scoping review found empirically supported models of practice from other countries where extensive work had been done or was under way in the relevant areas, including the Jed Foundation based in the USA, the Canadian Post-Secondary Mental Health Guide, the Under the Radar report from Orygen in Australia and Stepchange Mentally Healthy Universities in the UK. These recommended a whole system approach is needed to address student mental health and suicide prevention in higher education. Also, a consistent theme was that student mental health needs to be integrated through changes at all levels, ingrained into the campus culture, decisionmaking and participation across the whole sector and include all departments.

The literature also identified a gap, this framework is the first of its kind, there are no national higher education student mental health large-scale across whole sector or system policies or frameworks. The gap in the literature of national higher education student mental health policies suggests the development of a national-level policy may be necessary to create opportunities and environments, resources, endorsement, legitimization, and leadership systems at both subnational and national levels to encourage implementation and ultimately to impact in Ireland and beyond improvements in student mental health and wellbeing.

\subsection{International practice}

The Jed Foundation based in the USA is a nonprofit organization that has been instrumental in 
supporting student mental health. It takes a wholesystem approach that supports emotional health and reduces the risks of substance abuse and suicide. The primary mission of the foundation is to prevent suicide amongst students through promotion of awareness that 'mental illness is treatable and that suicide is preventable' [15]. The foundation provides action plans for campus mental health and protocols for responding to suicidal students.

From Canada we found the Post-Secondary Student Mental Health: Guide[16] that was developed in 2013 by the Canadian Association of College and University Student Services and the Canadian Mental Health Association. The guide aims to support the creation of third-level campus communities that are 'conducive to transformative learning and mental wellbeing'. The guide is designed as a resource to develop a whole-institution systemic approach to student mental health. Canada is similar to Ireland in that post-secondary institutions have independently developed policies and programs to respond to student mental health. The Mental Health Commission of Canada is currently holding a public consultation and a new standard is due to be released this year.

As in Ireland and Canada, universities across Australia have independently developed policies and programs to respond to student mental health and wellbeing. At a government level suicide prevention and mental health policies provide educational support only within primary and secondary schools. In 2017, Orygen, the National Centre for Excellence in Youth Mental Health, published Under the Radar: Mental Health of Australian University Students [17] with a view to:

- Improving data collection on student mental health

- Identifying the reasonable expectations of universities in responding to students' mental health issues

- Describing opportunities for partnership between universities and community mental health services

- $\quad$ Promoting the use of evidence-based, appropriate and acceptable programmes and interventions.

Leading on from the Under the Radar report, Orygen has been awarded funding to develop a National University Mental Health Framework that is due to be launched.

In 2017 Universities UK launched Stepchange: Mental Health in Higher Education [11], a framework for encouraging university leaders to adopt a strategic approach to the mental health of their populations. Remodeled and in May 2020 as Stepchange: Mentally Healthy Universities [18]. The primary aim of the framework is to offer guidance to HEIs on suicide prevention. Also, recently Student Minds launched The Universities Mental Health Charter [19]. The vision of the Charter is for all universities in the UK to become places where mental health and wellbeing are promoted for students and all members of the university community. The Charter has two main aims:

- 'Create an evidence-informed Charter that can provide a reference point for HEIs to adopt a wholesystem approach to mental health and inform ongoing enquiry and debate.

- Develop a Charter Award Scheme, which will assess HEIs against the Charter and recognise those providers who demonstrate excellent practice, providing further structure and building an evidence base which can inform ongoing improvement'.

\subsection{Models of Practice}

Zero Suicide [20] is a suicide prevention framework originally developed for clinical health care systems. The Zero Suicide framework is a multilevel view of suicide prevention with three core values: leadership, continual improvement and patient support. Zero Suicide is used widely in the US and is gaining traction internationally as an evidence-based suicide prevention model. HEIs in the US have adopted Zero Suicide prevention programs but for zero suicide to work in higher education there needs to be 'a hearts and minds shift - from anxieties about reputation, recruitment, duty of care and corporate responsibility to an overt focus on student suicide prevention' [21].

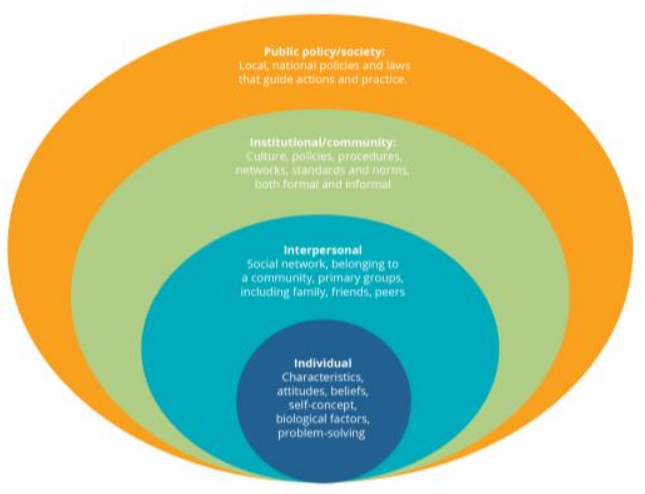

Figure 1. An ecological perspective on health promotion programs (adapted from McLeroy et al., 1988)

3.2.1. Social Ecological Model. It is clear that mental health needs to be integrated through institutional changes at all levels and into the campus culture, creating horizontal cooperation, decision-making and participation across the whole sector and include all 
departments. Improving and supporting student mental health requires a whole-system collaborative approach - this is not a job for a single department.

All of the examples of best practice to address student mental health and suicide prevention recognize the benefits of an embedded whole-system approach. The social-ecological model (see Figure 1) shows how behavior is influenced by the individual themselves and their environment. A comprehensive whole system mental health and suicide prevention framework needs to address all the levels of influence: public, institutional, interpersonal and individual.

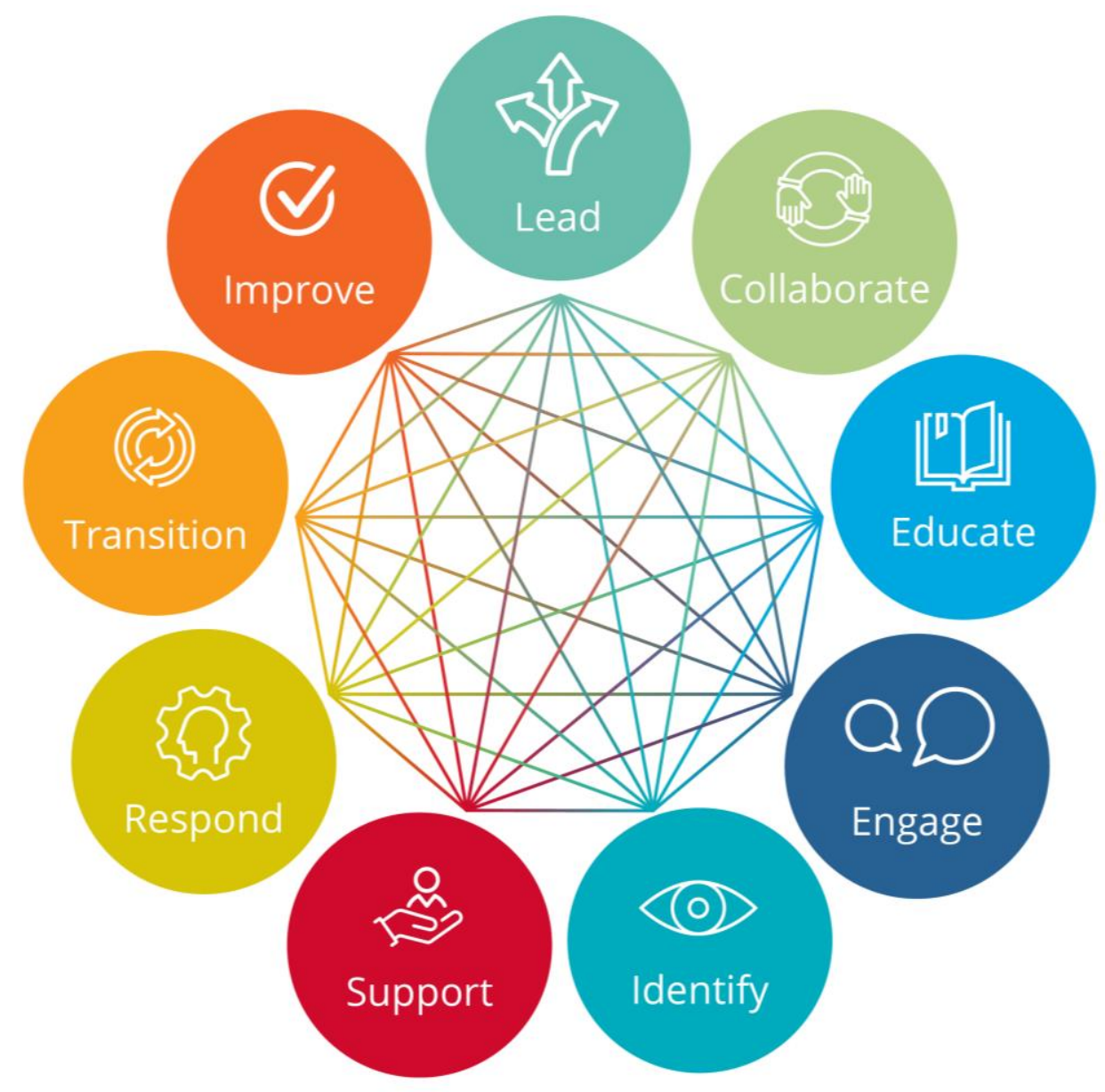

Figure 2. National Framework for Student Mental Health and Suicide Prevention

\section{Developing the Framework}

The Framework is Ireland's first national approach to address student mental health and suicide prevention. The national framework will help higher education institutes identify where further improvements for student mental health are needed, provide good practice guidance and resources, and help to standardize approaches across the country.

The framework, as shown in Figure 2, has nine interconnected themes: lead, collaborate, educate, engage, identify, support, respond, transition and improve. As discussed above developing a wholesystem collaborative approach for student mental health and suicide prevention - is not the responsibility of a single department nor is it the case for one theme.

\subsection{Lead}

Improvements in student mental health and suicide prevention will only be effective if they are led out and supported at a whole-sector level, through national policy and strategy. Regard for student wellbeing and mental health should permeate all aspects and be a key priority of decision-making at government level - this is necessary to ensure that HEIs can affect the changes necessary in their institutions. One way to ensure student mental health and suicide prevention remains on the agenda at sectoral and policy level is to establish an advisory 
group or committee on mental health in higher education.

Institution leaders need be the champions, so everyone at the whole institution knows what the goal for change is and understands the contribution they can make to the mission. Engagement and participation from all members of staff and students, including senior management, heads of departments and faculties, provosts, deans, student services professionals, student representatives, academic staff, tutors, students' unions, security and accommodation personnel will highlight mental health and remove obstacles for obtaining support.

\subsection{Collaborate}

HEIs need to support an inclusive co-creative approach where students and staff collaborate at every stage of the journey to improve mental health outcomes. HEIs also need to develop strong, highlevel and regular links with the national health service [HSE], with other local and national authorities, and with NGOs. They also need to develop strategies and action plans on student mental health and suicide prevention that are embedded not just throughout the HEI environment but also in the wider community. Some HEI student support services have established strong links with their regional HSE mental health services. Good communication with local mental health services will enhance access to treatment and support for students.

\subsection{Educate}

Education and training are key components to improving the nation's understanding of and attitudes to suicidal behavior, mental health and wellbeing [22]. Leading international experts have also stressed the importance of training community members to be gatekeepers who can identify signs of psychological distress and direct vulnerable students to appropriate resources [18], [19]. They also recommend that all student-facing staff (including academic, professional services and operational staff) across the whole organization, should be trained in mental health literacy and suicide awareness. The aim of the training is to promote mental health awareness, reduce mental health stigma and prevent suicide. Although the evidence on its long-term effectiveness is limited [23], [24], gatekeeper training is recognized as a systemwide preventive approach that can raise awareness, increase knowledge and build skills within the community [25], [26].

\subsection{Engage}

Encourage and foster the development of student wellbeing through community engagement, a sense of shared purpose, connectedness and belonging. Institutional culture needs to be inclusive and compassionate and reflect the diversity of the student body. Relationships are an important protective factor, including the sense of engaging with or being part of the institution, the wider campus community, and the student body Students with lower social support are at greater risk of depression and experience greater prevalence of suicidal thoughts [27]. In addition, promotion of healthy behaviors can have a significant impact on mental health [28]. Promoting good mental health on campus such as campaigns on: breaking down the stigma of mental health, tools for self-care, how to recognize the signs of being unwell, how to develop healthy coping strategies, and the importance of peer support. Some institutions provide students with mental health knowledge through academic courses [29] and their positive effects on students have been welldocumented [30]. Teaching students to recognize when they need support and where they can get it makes the idea of seeking support more normal and culturally acceptable.

\subsection{Identify}

Many HEIs in Ireland provide a wide range of mental health initiatives, including campaigns to raise mental health awareness and psycho-education for students and staff. Having 'regular and highly visible awareness raising interventions' [23] can help to establish a culture that supports students who may be experiencing poor mental health and help them identify appropriate services. Stepchange [18] recommend the creation of intrusive communities for learning and peer support where disclosure can be encouraged either through champions or through open, supported discussions. Intrusive communities for learning and support enable proactive interventions targeted at vulnerable or at-risk groups. Intrusive student support should include making nonacademic help an integral or mandated part of every student experience, and by so doing help make it easier for people to seek support [31].

\subsection{Support}

Ensuring access to effective, student-centered care, support and treatment is essential to the improvement and recovery of students experiencing mental health difficulties. As a priority, HEIs must provide students with high-quality services that offer evidence-based supports, interventions and therapies that are delivered by qualified professionals and that are subject to regular evaluation. On-campus counselling and health services require appropriate clinical governance, so they are safe, ethical and effective, and that they make efficient use of resources. This includes ensuring that staff in specific 
mental health roles are appropriately qualified, clinically supervised and registered with a professional body [26]. Measures to achieve these goals include:

- Provision of resources to match increased demand for services;

- Good internal and external communication;

- Cross-disciplinary and interdisciplinary collaborations with key stakeholders and access to referral pathways;

- Increased mental health and suicide risk screening to manage risk ;

- Data collection and information sharing systems contributing to a national standardised data set.

Just as students have different learning styles, the ways that they seek support also varies. For that reason, HEIs should provide students with multiple service access points for support, including in-person service as well as remote, online, text and telephone services, an after-hours service, access to group and peer support, and self-help resources.

\subsection{Respond}

Ensure that institutions have the critical incident protocols required for varying levels of student mental health crisis. A critical incident is an event out of the range of normal experience - one that is sudden and unexpected, where there is a loss of control, where there is a perceived threat to life, or where there are elements of physical and emotional loss [32]. Typically, critical incidents include fire, flooding, the outbreak of an infectious disease, and so on. In higher education, the death of a student by suicide must also be regarded as a critical incident, and HEIs need to respond appropriately to deal with the aftermath of such a sad and emotive event. HEIs should have an effective plan to follow, with defined members, a nominated lead person and agreed templates for communications [11], [33]. In the HEI community, where students often inhabit the same geographical area, social space and online space, a peer suicide can have a large ripple effect. It is widely accepted that good, coordinated suicide postvention has a critical role to play in the prevention of further suicidal actions in the community affected by the suicide [34].

\subsection{Transition}

Higher education is a time of transitions for students, they have to deal with independence, forming new relationships, dealing with financial independence, as well as with the pressures of academic exams and coursework. Starting college can be overwhelming and most HEIs hold an orientation events for first-year students, to connect them to each other and to the HEI. Certain groups and demographics are more susceptible to mental health difficulties at these times that might impact on their wellbeing and they need to be targeted with intrusive student support, appropriate initiatives and outreach programs at key transition points [23].

Students who are registered with mental health services may have additional stress and can experience significant disruption as they move from child and adolescent services to adult mental health services or to another service team nearer their new location [35]. It can mean an abrupt change in a student's level of responsibility and management of their mental health [36] and those receiving ongoing treatment may not be accustomed to organizing health care for themselves. These students would benefit from strong partnerships between universities support services and community mental health services and health passports [37] to continuity of care. Another significant yet under researched transition is the transition into final year. There is much advice available on exam stress but for final year students but there is the added pressure of their undergraduate experience coming to an end. Transition is a complex process of development, change and identity shifts. Higher education needs to move away from its narrow focus of transition initiatives and support for first-year students only [38] and recognize that students experience a series of transitions throughout their higher education journey [39].

\subsection{Improve}

Strategic and well-designed student mental health supports, and suicide prevention programs can only be developed after program planners have gained a thorough understanding of campus needs and how their programs are expected to achieve specified goals [40]. Conducting a baseline needs assessment and evaluation of current practices are key to developing the whole-system response for student mental health and suicide prevention. Ongoing audits, regular monitoring of plans and robust data collection systems drives an integrated, organization wide approach to improvement' [23]. Consider existing data collection mechanisms such as surveys on student satisfaction, retention, evaluation, quality indicators, wellbeing/life satisfaction measures, graduate outcomes surveys, counselling outcome measures, waiting times and various student safety and health indicators (for example, on violence, criminal activities, sexual assault and substance abuse). 


\section{Discussion}

Results from this study identified at present there is a lack of national-level policies on mental health and suicide prevention for higher education. The Irish National Student Mental Health and Suicide Prevention Framework for higher education is the first of its kind in Ireland and was launched by the HEA on world mental health day 10th October 2020. The framework is also cutting edge by encouraging adoption through its accompanied implementation guide [41], which will help higher education institutes identify where further improvements for student mental health are needed, provide good practice guidance, resources, tools and steps forward.

We have an area of future research to explore on national-level policy and gain understanding if institutional, community and national levels make a difference on outcome. There is also a body of future research on implementation, interventions, programs, curriculum, environments, resources, endorsement and leadership that are most effective.

This framework has potential to impact student mental health and wellbeing in Ireland and beyond. It emphasizes de-stigmatization of mental health and seeking support, building inclusive campus communities that are collaborative across sectors and disciplines, for improving student mental health, success in higher education and lifelong to a positive productive adulthood.

\section{References}

[1] Fox, T., Byrne, D., and Surdey, J., (2020) 'National Student Mental Health and Suicide Prevention Framework'; https://hea.ie/assets/uploads/2020/10/HEA-NSMHS-Frame work.pdf (Access Date: 10 November, 2020).

[2] Auerbach, R., Mortier, P., Bruffaerts, R., Alonso, J., Benjet, C., Cuijpers, P., Demyttenaere, K., Ebert, D., Green, J., Hasking, P., Murray, E., Nock, M., Pinder-Amaker, S., Sampson, N., Stein, D., Vilagut, G., Zaslavsky, A., Kessler, R., and WHO WMH-ICS Collaborators (2018). 'WHO World Mental Health Surveys International College Student Project: Prevalence and distribution of mental disorders', Journal of abnormal psychology, 127(7), pp. 623-638.

[3] Dooley, B., O'Connor, C., Fitzgerald, A. and O'Reilly, A. (2019). My World Survey 2: National Study of Youth Mental Health in Ireland, UCD and Jigsaw: Dublin.

[4] Dooley, B. and Fitzgerald, A. (2012). My World Survey: National Study of Youth Mental Health in Ireland. Headstrong: Dublin.

[5] Higher Education Authority (2019). 'Graduate Outcomes Survey: Class of 2018 HEA Report'; https://hea.ie/skills-engagement/graduate-outcomes/gradua te-outcomes-survey-data-and-results/ (Access Date: 10 November, 2020).
[6] Department of Health (2015). Connecting for Life: Ireland's National Strategy to Reduce Suicide 2015-2020. Department of Health: Dublin.

[7] Hyland, A. (2011). Entry to Higher Education in Ireland in the 21 st Century. NCCA-HEA, UCD: September.

[8] HEA, (2019) 'Graduates Outcomes Survey : Class of 2018'; https://hea.ie/assets/uploads/2017/11/HEA-Graduat e-Outcomes-Survey-Class-of-2018-1.pdf (Access Date: 10 November, 2020).

[9] TCD (2019). Annual Equality Monitoring Report 2018/2018. https://www.tcd.ie/equality/assets/docs/AEMR/ AEMR_2017-18_FullReport_Board.docx.pdf(Access Date: 10 November, 2020).

[10] Universities UK (2018). 'Suicide-Safer Universities'; https://www.universitiesuk.ac.uk/policy-and-analysis/repor ts/Documents/2018/guidance-for-sector-practitioners-on-pr eventing-student-suicides.PDF (Access Date: 10 November, 2020).

[11] OECD (2018). Health at a Glance: Europe 2018: State of Health in the EU Cycle. OECD Publishing: Paris.

[12] Kessler, R., Berglund, P., Demler, O., Jin, R., Merikangas, K. and Walters, E. (2005). Lifetime Prevalence and Age-of-Onset Distributions of DSM-IV Disorders in the National Comorbidity Survey Replication. Archives of General Psychiatry. 2005;62(6):593-602. doi:10.1001/arc hpsyc.62.6.593

[13] Higher Education Authority (2018). 'Key Facts and Figures Higher Education 2017/18'; https://hea.ie/assets /uploads/2019/01/Higher-Education-Authority-Key-FactsFigures-2017-18.pdf_(Access Date: 10 November, 2020).

[14] AHEAD (2019). Numbers of Students with Disabilities Studying in Higher Education in Ireland 2017/18. Association of Higher Education Access and Disability: Dublin.

[15] The Jed Foundation, (2020). 'What we do'; https://www.jedfoundation.org/what-we-do/ (Access Date: 10 November, 2020).

[16] CACUSS (2013). 'Post-Secondary Student Mental Health: Guide to a Systemic Approach'. Canadian Association of College and University Student Services and Canadian Mental Health Association Vancouver, BC: https://healthycampuses.ca/wp-content/uploads/2014/09/T he-National-Guide.pdf (Access Date: 10 November, 2020).

[17] Orygen (2017). Under the Radar: The Mental Health of Australian University Students. Orygen, The National Centre of Excellence in Youth Mental Health: Melbourne.

[18] Universities UK (2020). 'Stepchange: Mentally Healthy Universities' https://www.universitiesuk.ac.uk/step pchange -mhu (Access Date: 10 November, 2020).

[19] Hughes, G. and Spanner, L. (2019). The University Mental Health Charter. Leeds: Student Minds. 
[20] Zero Suicide (2020). 'About Zero Suicide'; http://zerosuicide.edc.org/about (Access Date: 10 November, 2020).

[21] Smith, J. and Howie, F. (2018). 'Preventing Suicide in Higher Education Institutions: Opportunities and Obstacles to Successful Suicide Prevention'. NSPA Conference, London; 31 January; www.nspa.org.uk/wp-content/uploads /2018/02/Preventing-student-suicide-in-higher-educationinstitutions-opportunities-and-obstacles-to-successful-suici de-preve ntion.pdf (Access Date: 10 November, 2020).

[22] National Office of Suicide Prevention (2019). The Education and Training Plan 2019-2020. NOSP: Dublin

[23] Dillon, L., Guiney, C., Farragher, L., McCarthy, A. and Long, J. (2015). Suicide Prevention: An Evidence Review. Health Research Board: Dublin.

[24] Isaac, M., Elias, B., Katz, L, Belik, S., Deane, F., Enns, M. and Swampy Creek Suicide Prevention Team (2009). Gatekeeper Training as a Preventative Intervention for Suicide: A Systematic Review. The Canadian Journal of Psychiatry, 54(4), 260-268.

[25] Mitchell, S., Kader, M., Darrow, S., Haggerty, M. and Keating, N. (2013). Evaluating Question, Persuade, Refer (QPR) Suicide Prevention Training in a College Setting. Journal of College Student Psychotherapy, 27(2), 138-148

[26] World Health Organization (2014). Preventing Suicide: A Global Imperative. World Health Organization: Geneva.

[27] Hefner, J. and Eisenberg, D. (2009). Social Support and Mental Health among College Students. American Journal of Orthopsychiatry, 79(4), pp. 491-499.

[28] World Health Organization (2005). Promoting Mental Health, Concepts, Emerging Evidence, Practice: Summary Report. World Health Organisation: Geneva.

[29] Lipka, S. (2010). Students Lose Weight for Credit. Chronicle of Higher Education, 57, A15.

[30] Mack, M. and Shaddox, L. (2004). Changes in ShortTerm Attitudes toward Physical Activity and Exercise of University Personal Wellness Students. College Student Journal, 38(4), pp. 587-593.

[31] Crisp, G. and Hatch, D. (Eds). (2016). Promising and High-Impact Practices: Student Success Programs in the Community College Context. Special issue of New Directions for Community Colleges. San Francisco, CA: Wiley Periodicals, Inc.

[32] World Health Organization, (2006). 'Stress Management in Emergency Deployment'; https://www.who .int/hac/techguidance/training/predeploment/Stress $\% 20 \mathrm{ma}$ nagement $\% 20$ in $\% 20$ emergency\%20development.pdf (Access Date: 10 November, 2020).

[33] Griffin, E. and McMahon, E. (2019). Suicide Bereavement Support: A Literature Review. National Suicide Research Foundation: Ireland.
[34] Andriessen, K., Rahman, B., Draper, B., Dudley, M. and Mitchell, P. (2017). Prevalence of Exposure to Suicide: A Meta-Analysis of Population-Based Studies. Journal of Psychiatric Research, 88, pp. 113-120.

[35] Student Minds (2017). 'Student Voices Report', Page 23; https://www.studentminds.org.uk/uploads/3/7/8/4/378458 4/170901_student_voices_report_final.pdf (Access Date: 10 November, 2020).

[36] Cleary, M., Walter, G. and Jackson, D. (2011). 'Not Always Smooth Sailing': Mental Health Issues Associated with the Transition from High School to College. Issues in Mental Health Nursing, 32(4), pp. 250-254.

[37] Thorley, C. (2017). 'Not by Degrees: Improving Student Mental Health in the UK's Universities'. Institute for Public Policy Research: London; https://www.ippr.org/ files/2017-09/1504645674_not-by-degrees-170905.pdf (Ac -cess Date: 10 November, 2020).

[38] O'Donnell, V., Kean, M. and Stevens, G. (2016). Student transition in higher education. Higher Education Academy.

[39] Morgan, M. (2011) 'Improving the Student Experience in Higher Education: Support and advice for staff'; http://www.improvingthestudentexperience.com/ (Access Date: 10 November, 2020).

[40] The Jed Foundation (2011). 'ULifeLine online resource for college mental health'; http://www.ulifeline.org/?_ga $=2.189347435 .1193273296 .1574857561-2007481454.156$ 5261945 (Access Date: 10 November, 2020).

[41] Fox, T., Byrne, D., and Surdey, J., (2020). 'National Student Mental Health and Suicide Prevention Framework: Implementation Guide'; https://hea.ie/assets/uploads/2020 /10/HEA-NSMHS-Framework-Implementation-Guide.pdf (Access Date: 10 November, 2020).

\section{Acknowledgements}

The authors of this research work are Treasa Fox (Project Lead), Dr Deirdre Byrne and Dr Jessica Surdey of the SynthSCS Project Team from Athlone Institute of Technology. The SynthSCS Project is funded by Higher Education Authority Innovation and Transformation Fund 2018. We thank our colleague from the HEA Connecting for Life Working Group and recognize their contribution and expertise that assisted the development the framework. SynthSCS is part of the 3SET Project a collaboration between Trinity College Dublin, University College Dublin and Athlone Institute of Technology that is addressing the increasing demand for student mental health support, Student Engagement and Success Strategy in Irish Higher Education. 